\title{
Immer unter Verdacht? - Identitätszuschreibungen im Kontext des Kleinhandels an der Außengrenze der Europäischen Union
}

\author{
J. Miggelbrink \\ Leibniz Institut für Länderkunde, Leipzig, Germany \\ Correspondence to: J. Miggelbrink (j_miggelbrink@ifl-leipzig.de)
}

Received: 31 July 2012 - Revised: 21 June 2013 - Accepted: 11 July 2013 - Published: 7 October 2013

Zusammenfassung. The harmonized, uniform Schengen border regime that secures the "Area of Freedom, Security and Justice" has led to remarkable changes of conditions for border crossing in Eastern Europe. Morover, its assemblage of documents, procedures, techniques, locations etc. of control and surveillance controls and governs the identities of travellers. The article analyses how small-scales traders at the Polish-Belarusian border, the Polish-Ukrainian border and the Romanian-Ukrainian border who operate on the edges of the legal and the formal (and even beyond) are being confronted with these new ascriptions of identities, the ways they feel being governed by the new border and their reactions to. The fieldwork focuses a period shortly after the Schengen border was shifted towards Eastern Europe in 2007.

\section{Einleitung}

Der Beitrag ${ }^{1}$ untersucht die Frage, wie Kleinhändler/innen an der östlichen Grenze des Schengenraums - speziell an den südlichen und östlichen Grenzen Polens zu Belarus und der Ukraine sowie an der Grenze Rumäniens zur Ukraine - sich selbst als Subjekte der Fremdwahrnehmung in der Ausübung des Grenzregimes reflektieren. Kleinhandel (zur Definition vgl. Abschnitt 2) ist eine verbreitete Tätigkeit in mittel- und osteuropäischen Grenzgebieten, die nach der politischen Wende ihren Höhepunkt erreichte (vgl. Haase et al., 2004:62ff.). Sie spielte und spielt in vielen Erwerbsbiographien im grenznahen Raum mindestens vorübergehend eine Rolle (Bruns, 2010b). Die informelle und illegale Tätigkeit hat oftmals eine kompensatorische Funktion in wirtschaftlichen Krisensituationen, indem sie an die Stelle nicht (mehr) vorhandener formaler Erwerbsmöglichkeiten und dysfunktionaler, fehlender oder unzureichender sozialer Sicherungssysteme tritt (Wagner, 2010:88f). Im Unterschied zu manchen anderen grenzüberschreitenden Tätigkei-

\footnotetext{
${ }^{1}$ Ich danke Christoph Waack, Frank Meyer sowie drei anonymen Gutachter/innen für ihre konstruktiven Hinweise zu einer früheren Fassung dieses Beitrags.
}

ten ist die Grenze im Kleinhandel nicht nur ein zu überwindendes Hindernis, sondern zugleich dessen zentrale Ressource, weil er auf der Möglichkeit basiert, durch die Ausnutzung von Preis-, Angebots- und Nachfragedifferenzen zwischen benachbarten Nationalökonomien Gewinne zu erzielen. Die Bedingungen, unter denen das ,resourcing borderlands" (Weiss, 2012:213) stattfindet, haben sich in den letzten Jahren jedoch gewandelt: Seit Dezember 2007 müssen Reisende aus Drittstaaten, die nach Polen einreisen wollen, über ein Schengen-Visum verfügen, das gebührenpflichtig ist und persönlich bei den zuständigen Konsulaten beantragt und abgeholt werden muss. Reisende nach Rumänien und Bulgarien müssen, da das Schengen-Visum in Rumänien noch nicht ausgegeben wird, ein nationales Visum beantragen, dessen Vergabebedingungen denen des SchengenVisums angeglichen ist ${ }^{2}$. Da der Erwerb des nationalen Visums mit einem dem Schengen-Visum vergleichbaren Aufwand für die Reisenden verbunden ist, wird es aus der Perspektive der Reisenden als Teil des Schengen-Regimes wahrgenommen. Zwar gab es auch vorher schon zahlreiche Formen der Kontrolle grenzüberschreitender Bewegungen (Migrationskarten, Sichtvermerke in Pässen), diese konnten aber

\footnotetext{
${ }^{2}$ Polen stellt zusätzlich ebenfalls nationale Visa aus.
} 
mit weniger Aufwand an Zeit, Kosten, Mühe und Kontrolle beschafft werden.

Ein wichtiges Moment im Kalkül der Kleinhändler/innen ist stets das Wissen darum, unter welchen Voraussetzungen die Grenze überquert werden kann bzw. wann und wo diese Bedingungen günstig für das eigene Handeln sind. Das betrifft sowohl formale, regulatorische Aspekte (Visum, Deklarationen) als auch praktische (wann und wo wird schnell abgefertigt, welche Grenzschutzbeamte sind kulant, welche Zöllner/innen evtl. bestechlich, wo finden regelmäßig Hinterlandkontrollen statt). Nicht unwesentlich, in der Literatur aber bislang weniger behandelt, ist die Frage, wie „man“ an der Grenze behandelt wird. Jedes Grenzregime enthält einen Komplex von Zuschreibungen, der die Identität der Reisenden und die Form ihrer Behandlung betrifft, denn jede Grenze entfaltet eine strategische Selektivität, mittels derer die „unerwünschten Eintritte“ (Andreas, 2003:80) von den erwünschten getrennt werden sollen. Diese ist nicht allein durch den politischen Willen zur Regulierung definiert, auch nicht nur durch die Art und Effizienz der eingesetzten Techniken zur Identifikation, Filterung und Sortierung der (potenziellen) Grenzüberquerer. Ihre Bedeutung konkretisiert sich vielmehr erst im Moment der Grenzüberquerung, wenn staatliche bzw. suprastaatliche Souveränität und Gesellschaft in Form sozialer Beziehungen, alltäglicher Interaktionen und Kommunikationen aufeinander treffen und die Grenze zu einem Medium wird ,through which institutions, associations or legal machineries operate“ (Nadel, 1956:171f. zit. n. Donnan und Wilson, 2003:11, Herv. JM).

Gegenstand dieses Beitrags sind die auf das Subjekt bezogenen Strukturierungsansprüche des Schengener Grenzregimes, durch die das Feld legitimer Identitäten, Subjektpositionen und Praktiken formiert wird (vgl. Laclau und Mouffe, 1985:115f.; Davies und Harre, 1990). Strukturierungsansprüche gehen vielfach von staatlichen bzw. politischen Institutionen aus, die - weil sie als legitime Pole der Verfügung über Gewalt und Hoheit über das Gesetz gelten auch eine Definitionshoheit über bestimmte soziale Kategorisierungen und deren Kontrolle haben (z.B. kriminell, Terrorist, Ehe). Laclau und Mouffe (2006) folgend, nutzen politische Projekte das Strukturieren der semantischen Sphäre - das Anordnen von Signifikanten zueinander (vgl. Torfing, 2005:14f.) - dazu, Legitimes von Illegitimem zu trennen. Indem durch solche Strukturierungsakte Identitäten als mögliche Subjektpositionen definiert werden (ebd., 15), wird eine Art soziale Struktur diskursiv erschaffen. Innerhalb dieser Strukturierung positionieren sich die Subjekte und passen sich ggf. daran an (siehe z.B. Bröckling, 2007). In Grenzregimes werden insofern souveräne Strukturierungsansprüche manifest, als sie die erwünschten und legitimen von den unerwünschten und nicht-legitimen Grenzüberschreitungen unterscheiden und dafür Kategorien, Zuschreibungen, Mechanismen und Prozeduren der Sortierung und Filterung entwickeln müssen. Im Kontext des Schengener Grenzregimes ist, wie unten ausgeführt wird, die Kategorie der staatsbür- gerschaftlichen Zugehörigkeit und die geopolitische Positionierung des Herkunftslandes ein zentrales, aber nicht das einzige Mittel der Strukturierung. Mechanismen und Prozeduren, wie die Beantragung und Ausstellung von Visa, die Offenlegung der Reisemotive, das Erheben von Gebühren usw. sind Akte der Strukturierung, die sowohl auf das Individuum wirken als auch die Wahrnehmungsschemata der Grenzbehörden prägen. Die daraus resultierenden Formierungen werden hier in zweierlei Hinsicht thematisiert: Zum einen als Effekte eines zunehmend ,versicherheitlichten“ Grenzregimes (vgl. Belina und Miggelbrink, 2013), in dem die Vergabe von Visa ein zentrales Instrument einer vorgelagerten, auf ganze Bevölkerungen wie auf Individuen bezogenen Kontrolle ist. Zum anderen als Folgen der praktischen Ausübung dieses Grenzregimes. Während Ersteres theoretisch hergeleitet werden kann (vgl. Abschnitt 3), basiert Letzteres auf empirischen Untersuchungen (Abschnitt 4 und 5), die im Rahmen des Projekts „Geographie(n) an den Rändern des europäischen Projekts" im Frühjahr und Sommer 2008 in Tohmajärvi (Finnland), Sortavala (Russland), Białystok und Przemyśl (Polen), Schowkwa und Solotvino (Ukraine) sowie in Sighetu Marmatiei (Rumänien) durchgeführt wurden.

\section{Grenzüberschreitender Kleinhandel als soziale Praxis}

Kleinhandel ist als ökonomische Tätigkeit nicht klar definiert (vgl. Bruns, 2010a; Wagner, 2010; Polese, 2012). Zumeist wird darunter verstanden, dass auf eigene Rechnung arbeitende Personen kleinere Mengen an Waren - in den hier untersuchten Beispielen an der polnisch-belarussischen, der polnisch-ukrainischen sowie der rumänisch-ukrainischen Grenze handelte es sich um Zigaretten, Alkohol, Kleidung (inkl. Schuhe), Hygiene- und Reinigungsmittel sowie elektronische Geräte, seltener um Lebensmittel - auf der einen Seite der Grenze einkaufen, um sie auf der anderen Seite wieder zu verkaufen. Sie verfügen meist nur über geringes Eigenkapital, arbeiten allein oder in Netzwerken aus Familienangehörigen und Bekannten, die entweder ",mithelfend“ tätig sind oder - gegen ein Entgelt - für bestimmte Tätigkeiten angeheuert werden, etwa wenn eine bestimmte Warenmenge an der Grenze auf mehrere Personen aufgeteilt werden muss, um den Zollbestimmungen Genüge zu tun. Weil im Kleinhandel Gewinne oft erst dadurch möglich werden, dass Einfuhrbestimmungen und Verzollung umgegangen werden - indem Waren versteckt werden, weil sie legaler Weise nicht gehandelt werden dürfen, oder indem Waren, die zum Verkauf bestimmt sind, als Eigenbedarf deklariert werden - findet er zumeist in der Grauzone zur Informalität und Illegalität statt (vgl. Bruns et al., 2011:666ff.; Castells und Portes, 1989:13). Zwar lassen sich Verstöße gegen bestehende Reglementierungen meist recht klar benennen, in der Praxis der Kleinhändler/innen sind jedoch legale Handelsaktivitäten und die ,illegale Tätigkeit“ (Bruns, 2010a:74) 


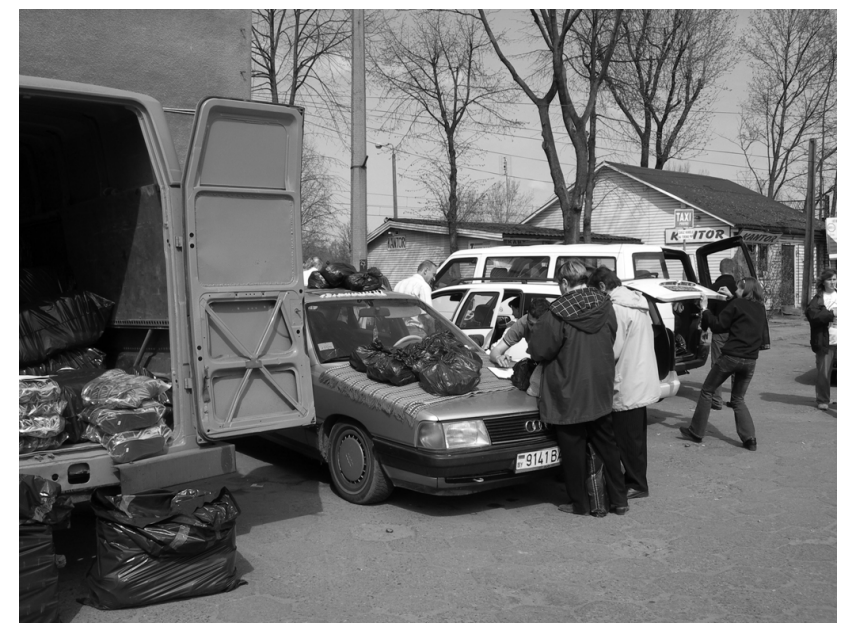

Abbildung 1. Kleinhändler/innen in Grodno/Belarus (Foto: Kristine Müller, 2007).

des Schmuggels nicht immer scharf zu trennen (Round et al., 2008:172). Daher plädieren Smith und Stenning zu Recht dafür, informelle und formelle ökonomische Aktivitäten nicht als ,separate oder abstrahierte ökonomische Formen zu betrachten, sondern (...) als miteinander verwobene ökonomische Praktiken" (Smith und Stenning, 2006:192). Neben juristischen Aspekten von Informalität und Illegalität spielen in der Praxis des Kleinhandels auch andere, i.w.S. moralische Kategorien eine Rolle (vgl. Bruns und Zichner, 2010): So kann das illegale Tun subjektiv wie kollektiv als gerechtfertigt und legitim angesehen werden, weil es in Bezug auf die Sicherung des Lebensunterhalts oder der sozialen Stabilität einer Region als alternativlos erscheint. Ebenso kann der Umgang staatlicher Instanzen mit dem Kleinhandel von der Duldung bis zur aktiven Förderung (vgl. Bruns) die Grenzen des Legitimen und sogar, wenn bestehendes Recht nicht durchgesetzt oder praktische Verstöße legitimiert werden, die Grenzen des Legalen verschieben. Kleinhändler/innen ziehen - nicht zuletzt zur Legitimierung ihres eigenen harmlosen Tuns - eine oft nicht genau zu bestimmende Grenze zum ,richtigen“, d.h. ,großmaßstäbigen“ Schmuggel, den sie ablehnen. Jenseits der juristischen Einordnung, im temporären Netz des alltäglichen Handelns und Redens (vgl. Schatzki, 2002:87), ist vor allem das Beherrschen der inoffiziellen, gleichwohl meist fest etablierten Spielregeln relevant, die die Händler/innen ebenso wie die Vertreter/innen von Zoll und Grenzschutz kennen müssen (vgl. Stammler-Gossmann, 2012:244ff.). Wie erfolgreich grenzüberschreitend agierende Kleinhändler/innen sind, hängt davon ab, ob sie die Taktiken der Subversion beherrschen, und zwar innerhalb der sozial produzierten und daher relationalen kategorialen Ordnungen, die ihr Tun strukturieren und auf die es zurückwirkt.

Auf wesentliche Bedingungen der Strukturierung dieser Handlungsmöglichkeiten haben Kleinhändler/innen jedoch zumeist keinen Einfluss. Das betrifft unter anderem die nor- mative Bewertung von Kleinhandel durch Grenzreglementierungsdiskurse. Diese hat sich - in erster Linie durch die Tätigkeit der europäischen Grenzschutzagentur FRONTEX ${ }^{3}-$ verschoben von einer weithin ignorierten Tätigkeit hin zu einer Bewertung als ,low risk - high profit“-Tätigkeit (FRONTEX, 2011a:10), die eine ,physical security thread“ darstellt und als ,schwere organisierte Kriminalität" eingeordnet wird (Laitinen, 2011:2, 12). Eine weitere Strukturierung entsteht aus dem zentralen Instrument der Versicherheitlichung der Schengen-Grenze, dem Schengen-Visum, dessen Effekte in Bezug auf die geopolitische wie individuelle Wertung ,des Anderen“ im folgenden Abschnitt skizziert wird.

\section{Die Funktion des Schengen-Visums im versicherheitlichten Grenzregime}

Das Schengener Vertragswerk, die zentrale Voraussetzung zur europäischen ökonomischen Binnenintegration, geht seit Mitte der 1980er Jahre mit einer „Neuordnung der Beziehungen zwischen den Nationalstaaten und einer partiellen Transnationalisierung staatlicher Aktivitäten" (Belina und Miggelbrink, 2010:225) einher, durch die die konstitutive Beziehung zwischen Territorium, Nationalstaat und Souveränität zwar nicht aufgehoben, aber doch ihrer Selbstverständlichkeit entkleidet wurde (Balibar, 2009:192). In Bezug auf die Bevölkerung des neuen territorialen Gebildes äußert sich diese Reskalierung von Souveränität im Entstehen des europäischen Bürgers als ,neue historische Figur“ (ebd., 202). Seit dem Vertrag von Amsterdam (1999) wird die neue territoriale Form als „Raum der Freiheit, der Sicherheit und des Rechts“ bezeichnet und durch die Europäische Sicherheitsstrategie (Ein sicheres Europa 2003) von ihrem Äußeren gleichzeitig abgegrenzt und mit ihm verschränkt (vgl. Van Houtum und Pijpers, 2007). Damit zeichnet sich auch eine neue Ordnung des „Fremden“ ab: Während die Unionsbürger/innen, zumindest auf der Ebene kategorialer Ordnung, einander etwas weniger fremd werden, verschärft sich die Grenze nach außen zu den „Drittstaatangehörigen“ (ebd., 203f.). Diese Neuordnung ist Teil einer zunehmenden Versicherheitlichung, mittels derer - in Anlehnung an das Dispositiv der Sicherheit (Foucault, 2007/1977-1978:19, 39ff.) - „Ereignisse und potenzielle Ereignisse, Institutionen und Maßnahmen, Aktivitäten und Personen ... in ein Szenario der Bedrohung und eines daraus resultierenden Bedürfnisses nach Überwachung, Kontrolle und Prävention" eingeordnet werden (Belina und Miggelbrink, 2013:125, Übersetzung JM) ${ }^{4}$. In Bezug auf den Personenverkehr, der in diesem Beitrag von besonderem

${ }^{3} \mathrm{Zu}$ Aufgaben und Funktion vgl. Fischer-Lescano und Tohidipur (2007) und de Boer (2010).

${ }^{4}$ Das ist im Kontext der europäischen Integration an sich kein neues Phänomen (vgl. Huysmans, 2000, für den Zeitraum nach 9/11 Boswell, 2007; Neal, 2009). Auch ist die Herstellung von Sicherheit, die die Abwehr von Terroristen ebenso einschließt wie die Verteidigung interner Wohlfahrt, keineswegs exklusiv Aufgabe der grenzbezogenen Politiken. Diesen fällt aber insofern eine zentrale 
Interesse ist, lassen sich gegenwärtig mindestens drei Formen einer versicherheitlichten Einordnung von Personen beobachten: Zum einen wurde und wird das Feld der Migration, wie Huysmans (2000) gezeigt hat, versicherheitlicht, indem Migration in der öffentlichen Wahrnehmung zunehmend als „Gefahr für die öffentliche Ordnung, kulturelle Identität sowie der Stabilität des Binnen- und Arbeitsmarktes“ (Huysmans, 2000:752) präsentiert wird. Aus diesen diskursiven Rahmungen resultieren nicht nur vielfach negative und ablehnende Wertungen von Migration, durch die Migration oftmals mit illegaler Migration gleichgesetzt wird, sondern auch entsprechende Praktiken des Umgangs mit Migration und Migrierenden. Indem die Annahme einer Gefährdung des „Inneren“ durch Migration als faktische Bedrohung gehandhabt wird, die die Durchsetzung strikter präventiver Maßnahmen notwendig und zwingend erscheinen lässt, wird Migration zu etwas, das es zu verhindern gilt. Versicherheitlichung erschwert folglich die Inklusion und Unterstützung von Migrierenden, Asyl-Suchenden und Flüchtlingen (ebd., 752). Zweitens haben sich Diskurse und Praktiken der Grenzkontrolle nicht nur in den USA als Teil der Herausbildung eines ,,post-September 11 security environment" (Andreas, 2003:94) gewandelt, sondern auch in Europa. Die Notwendigkeit der Abwehr terroristischer Akte ist als Legitimation grenzsichernder Maßnahmen deutlich in den Vordergrund getreten. Einen dritten Strang bildet der Topos der organisierten Kriminalität (einschließlich Menschenhandel). In zahlreichen Dokumenten der Grenzschutzagentur FRONTEX werden diese drei Stränge eng zu einem Konglomerat von Phänomenen zusammengeführt, die sie insgesamt als Bedrohung des Inneren der EU erscheinen lässt (vgl. z.B. FRONTEX, 2011a und b, 2012). Dadurch gewinnt die Projektion abstrakter Bedrohungen auf individuelle Reisende mit den Mitteln scheinbar objektiver Risikokalkulation an Bedeutung, als deren Folge jeder ,gewöhnliche Mensch“ latent kriminalisiert wird.

Das einheitliche Schengen-Visum ist eins der zentralen Instrumente im Prozess der Versicherheitlichung, weil mittels der Visavergabe jeder Reisende anlässlich jeder Rei$\mathrm{se}^{5}$ individuell beurteilt wird. Die Vergabe des Visums erfolgt durch die Konsulate der Schengen-Mitgliedstaaten; es setzt eine persönliche Abgabe des Antrags, eine Prüfung des Antragsstellers durch die Mitarbeiter des Konsulats sowie eine Abfrage in den Datenbanken des SchengenInformationssystems voraus. Auf diese Weise wird nicht nur ein wesentlicher Teil der Prüfung der Einreiseberechtigung von den Vertretern des Grenzschutzes an den Grenzübergangsstellen auf vorgelagerte Behörden verschoben, vielmehr sollen Reisewillige systematischer und intensiver über-

Rolle zu, als sie die geeigneten Maßnahmen entwickeln müssen, um - unter der Prämisse der Garantie der jeweils als relevant erachteten Form von „Sicherheit“ - die erwünschten von den unerwünschten Grenzüberschreitungen unterscheiden zu können.

${ }^{5}$ Oder, im Falle des Mehrfachvisums, einer Anzahl von Reisen innerhalb eines bestimmten Zeitraums. prüft werden. Insofern ist das Visum Teil eines Prozesses der „Ausweitung und Vorverlagerung sozialer Kontrolle" (Singelnstein und Stolle, 2012:122, Herv. JM) ${ }^{6}$. Mit ihm wird eine zweifache Zuschreibung von Identität vorgenommen: In einem ersten Schritt unterscheidet das VisaRegime mittels staatsbürgerschaftlicher Zuordnung Reisende aus visumspflichtigen Staaten von solchen aus nichtvisumpflichtigen Staaten. Das Individuum wird auf diese Weise anhand des ,geopolitischen Images“" seines Herkunftslandes positioniert (vgl. Bekus-Goncharova, 2008), indem individuelle (Einreise-) Rechte an geopolitisch stratifizierte Rechte gekoppelt werden (vgl. Buckel und Wissel, 2009). Daran schließt sich dann mit der konkreten Vergabe bzw. Verweigerung eine auf das Individuum bezogene Inklusions- und Exklusionstechnik an. Weil das SchengenVisum sowohl Ausdruck der Bewertung des Herkunftslands als auch ein Nachweis der Prüfung der Vertrauenswürdigkeit des/der einzelnen Reisenden mittels der von den Konsulaten der Schengen-Staaten durchgeführten Prüfungsverfahren (u.a. Datenbankabfragen) ist, entsteht eine Situation, die als kollektive Abwertung verstanden werden kann: „Nicht mehr dem Pass der Herkunftsländer (...) soll bei der Identifizierung von Reisenden aus Drittstaaten vertraut werden, sondern primär dem von Konsulaten der EU-Staaten ausgestellten Visum“ (Töpfer, 2008:[4]). Anders als zusätzlich zum gültigen Reisedokument mitzuführenden Migrationskarten oder das Stempeln von Pässen, die lediglich das Überschreiten der Grenze festhielten und bestätigten, ist das Visum eine auf das Individuum bezogene Einreiseerlaubnis, die bestätigt, dass diese Person die spezifischen Einreisevoraussetzungen des Ziellandes erfüllt. Die geopolitische Sortierung verbunden mit Entwertung des eigenen Herkunftslands in Bezug auf dessen (souveränes) Recht international akzeptierte Reisedokumente auszustellen, machen das Schengen-Visum zum ,Zwangsmittel externer Identifikation" (Brubaker und Cooper, 2000:1), zu einem Instrument der Fremdzuschreibung von (geopolitischer) Identität, das sich fundamental von jeder Selbstidentifikation unterscheidet und praktisch zu einem ,Gefühl der Diskrepanz, Geringschätzung und Erniedrigung“ (ebd.) führt, wie in Abschnitt 5 gezeigt wird. Die Schengen-Grenze wirkt nicht mehr nur als geopolitisches, sondern auch als biopolitisches Instrument, wie Walters feststellt (2002:571 et passim), indem sie zunehmend zum ,systematischen Instrument der Kontrolle nationaler und transnationaler Bevölkerungen“" (ebd., Übersetzung JM) wird, das - realisiert durch ein Ensemble diskursiver und nicht-diskursiver Praktiken (ebd., 572) - mit einer granularen Verfeinerung der Methoden und Techniken

\footnotetext{
${ }^{6}$ Besonders einschneidend haben sich die individuellen Reisemöglichkeiten aus der Sicht derer verändert, die zuvor lediglich an der Grenze einen Reisepass und ggf. eine in der Regel leicht zu erlangende Migrationskarte vorzeigen mussten (teils mit zusätzlichem Stempelverfahren), wie beispielsweise zwischen Polen und Belarus bzw. Polen und der Ukraine.
} 
der Kontrolle, Überwachung, Sortierung und Bewertung der Subjekte einhergeht.

\section{Orte und Zeitraum der empirischen Untersuchungen und angewandte Methoden}

Im einleitend genannten Forschungsprojekt (2007-2009) wurden im Wesentlichen zwei methodische Verfahren angewandt: Gruppendiskussionen, jeweils mit 5-10 Kleinhändler/inne/n, sowie ergänzend qualitative Interviews mit einzelnen Händler/inne $/ \mathbf{n}^{7}$ und Vertreter/inne/n des Zolls und der Grenzschutzbehörden. Während die Diskussionen und Gespräche mit den Händler/inne/n größtenteils aufgezeichnet werden konnten (mit anschließender Transkription und Übersetzung), konnten von den Gesprächen mit Grenzkontrollbehörden nur Gedächtnisprotokolle angefertigt werden. An den Diskussionen nahmen in etwa gleich viele Frauen und Männer überwiegend mittleren Alters teil, die alle seit Längerem, einige aber nur nebenberuflich, gelegentlich oder sporadisch im Kleinhandel aktiv sind. Die Geschlechts- und Altersverteilung entsprach nicht ganz unseren eigenen Beobachtungen sowie den Aussagen in den Gesprächen, dass Kleinhandel eher von Frauen als von Männern betrieben wird und dass häufig Rentner/innen involviert sind. Die Methode der Gruppendiskussion wurde gewählt, um Situationen des Erzählens und des Austausches zu schaffen, in denen die anwesenden Wissenschaftler - anders als im Interview idealerweise möglichst in den Hintergrund $\operatorname{treten}^{8}$, während die Teilnehmer/innen sich im Austausch von Erfahrungswissen über kollektive und ggf. davon abweichende individuelle Deutungen und Orientierungsmuster verständigen (zur Methode allgemein vgl. u.a. Bohnsack (1999), zum Gebrauch im Rahmen des Forschungsprojekts sowie den damit verbundenen Schwierigkeiten (Müller und Wust, 2013). Das transkribierte und übersetzte Material wurde unter Verwendung der Software MaxQDA inhaltsanalytisch ausgewertet. Aus den teils deduktiv aus den Ausgangsfragen des Projekts und den Impulssetzungen für die Diskussionen abgeleiteten, teils induktiv aus der Sichtung der Transskripte gewonnenen Codes wurden für diesen Beitrag in erster Linie jene Codings ausgewertet, die dem „Wie?“ der Praktiken des Grenzübertritts zugeordnet wurden, da in diesen Codings zahlreiche Aussagen darüber enthalten sind, wie sich die Betroffenen von

\footnotetext{
${ }^{7}$ In Belarus konnte aufgrund der Angst der Kleinhändler/innen vor Überwachung und anschließenden Repressionen keine Gruppendiskussion stattfinden. Hier waren lediglich Einzelinterviews an einem als „sicher“ erachteten Ort - ein Café - möglich.

${ }^{8}$ Da der Anlass zur Gruppendiskussion sowie die Bedingungen, unter denen diese stattfanden, von Seiten der Wissenschaftler/innen gesetzt wurden, und diese anwesend waren, auch wenn sie die Diskussion nicht selbst moderierten, handelte es sich nur um ein graduelles In-den-Hintergrund-Treten. Die artifizielle Situation der Gruppendiskussion erzeugte ebenso wie das Interview Erwartungen und Vorstellungen bei den Teilnehmer/inne/n, die das Gesprächsverhalten und den Verlauf beeinflussen.
}

anderen wahrgenommen und positioniert sahen. Ergänzend wurden einige Aussagen aus den Gruppendiskussionen und Interviews mit Kleinunternehmer/inne/n aus dem Dienstleistungsbereich und dem produzierenden Sektor herangezogen, deren grenzüberschreitende Praktiken im Rahmen des Projekts ebenfalls untersucht, hier aber nicht näher dargestellt werden (vgl. Bruns et al., 2010; Müller, 2013). Im weiteren Verlauf des Textes verwende ich den Begriff Kleinhändler/in für den erst genannten Personenkreis, den Begriff Kleinunternehmer/in - der genau genommen die Kleinhändler/innen einschließen würde - für Personen, die in anderen Branchen tätig sind.

Das aus heutiger Sicht nicht mehr ganz junge Datenmaterial ist insofern aufschlussreich, als es zu einem Zeitpunkt entstand, an dem die vollständige Umsetzung des Schengener Acquis an den polnischen Grenzen zu Belarus und der Ukraine erst wenige Monate zurück lag (Dezember 2007) und die Gesprächspartner/innen daher unmittelbar das Vorher und Nachher aus eigener Erfahrung miteinander vergleichen konnten. In diesem Zeitraum wurde auch die „baldige“, jedoch mehrfach verschobene vollständige Umsetzung des Acquis an den rumänischen Grenzen zur Ukraine und zur Republik Moldau in den Medien wiederholt thematisiert und beschäftigte v.a. unsere ukrainischen Gesprächspartner/innen sehr. Aus dem Material werden im Folgenden einige Identitätszuschreibungen diskutiert, mit denen sich die Gesprächsteilnehmer/innen im Rahmen ihrer kleinhändlerischen Tätigkeit konfrontiert sahen

\section{Subjekte an der Grenze}

\section{1 „Ihr seid Brüder und wir sind Brüder"}

Die oben abstrakt beschriebene Differenz zwischen der Europäischen Union, dem Schengenraum bzw. dem „Raum der Freiheit, der Sicherheit und des Rechts“ auf der einen Seite und deren dadurch konstituierten Außen auf der anderen äußert sich alltagspraktisch in erster Linie in den erschwerten Reisebedingungen. Symbolisch wird sie als ,große chinesische Mauer" (Cristi Creanga, rumänischer Unternehmer, GD 6, Z. 1297), als ,neue Berliner Mauer“ (Dariusz, ukrainischer Unternehmer, ehemaliger Kleinhändler, GD 7, Z. 214) und als ,richtig große Mauer“ (Arkadi, polnischer Kleinhändler aus Przemyśl, GD 4, Z. 1061) beschrieben. Die dadurch erzeugte Exklusion wird von vielen Reisenden jedoch nicht oder nicht ausschließlich als politische gesehen, sondern als ökonomische bzw. als eine politische, die mit ökonomischen Mitteln erzwungen wird, denn (nur) wer über ausreichend Geld verfügt, kann sich das zum Erwerb eines Schengen-Visums notwendige Prozedere leisten. Dass die Grenze über ihre geopolitische Rolle hinaus auch eine biopolitische Funktion hat, die auf die Herstellung einer internen Bevölkerung gegenüber einer (diffusen) äußeren zielt, bringt, quittiert mit dem Lachen aller, ein ukrainischer Kleinhändler zum Ausdruck: „Alle Menschen sind Brüder - nicht 
mehr [lacht]. Ihr seid Brüder und wir sind Brüder" (GD 1, Z. 1134f.).

Nicht nur die Spaltung zwischen Unionsbürgerschaft und Drittstaatszugehörigkeit, die sich aus der Sicht der Reisenden im Schengen-Visum manifestiert, beschäftigt die Gesprächsteilnehmer/innen, sondern auch die Verlagerung der auf die Person bezogenen Beurteilung, die als anmaßend empfunden wird. So empörte sich Basarab, ein ukrainischer Kleinhändler aus Solotvino nahe der rumänischen Grenze, darüber von einer fremden Macht beurteilt zu werden: „Man hat einen internationalen Reisepass - wer kontrolliert einen besser als die eigenen Leute? Niemand aus Europa kontrolliert, was ich mache, wovon ich lebe, wovon ich atme, so gut wie unsere Behörden hier vor Ort. Sie führen eine Kontrolle durch können sie mich ins Ausland lassen oder nicht?" (GD 1, Z. 1649-1953).

\section{2 "Menschen dritter Klasse" und nationale Stereotype}

Während die kategoriale Ordnung von Innen und Außen eine kollektive, auf Bevölkerungen gerichtete Dimension hat, ist der Akt der Grenzüberquerung ein Moment subjektiver Identifikation, in der die Konfrontation von Staatsmacht und Individuum in eine Interaktion übersetzt wird, die einerseits deutlich hierarchisiert ist, andererseits aber auch zahlreiche kontextuelle Bezüge enthält. Der Grenzübertritt ist stets ein besonderer Moment, der, - wie unsere Gesprächsteilnehmer/innen immer wieder betonten, „Demut“ erfordert und oft „demütigend“ ist. Ebenso betonten einige Gesprächsteilnehmer/innen, dass sie eine „schlechte Behandlung“ erwarten, diese aber einen bestimmten Punkt nicht überschreiten dürfe. Gerade die „schlechte“ (langsame, erniedrigende) Behandlung von ukrainischen Reisenden durch die polnischen Grenzautoritäten ist ein immer wiederkehrender Topos. Ukrainer würden, wie ein polnischer Unternehmer meinte, oft als „Menschen dritter Klasse“ (GD 4, Z. 424) behandelt. Ein polnischer Unternehmer aus Przemyśl, Artur, sieht das so, dass die polnische Seite - Grenzschutz und Zoll - „oft die EU als eine persönliche spanische Wand benutzt - , weil die EU das verlangt“" (GD 7, Z. 1562f.). Das läge, so vermuteten einige Gesprächsteilnehmer/innen, am mangelnden Interesse an ukrainischen Reisenden und allgemein an nationalen Stereotypen. Eine stärker quantitativ ausgerichtete Studie der Batory-Foundation (2008:60 et passim) bestätigt zwar die Beobachtungen zur schlechten Behandlung, kann aber keine eindeutigen Gründe identifizieren.

Praktisch äußert sich die herabsetzende Behandlung durch Grenzautoritäten für viele Reisende v.a. im Geduzt-Werden. Manchmal, so räumt Artur ein, sei das ein Versehen, aber „manche, so scheint es mir, gehen da so ran“(ebd., Z. 448f.). Für ihn ist es offen, ob dies Ausdruck der Überlegenheit der Vertreter/innen der Grenzautoritäten qua Amt ist, ob es sich um die gängige schlechte Behandlung von Ukrainer/inne/n handelt oder ob es eine Haltung ist, die sie sich angesichts der Vielzahl von Schmuggler/inne/n zu gelegt haben. Ob-

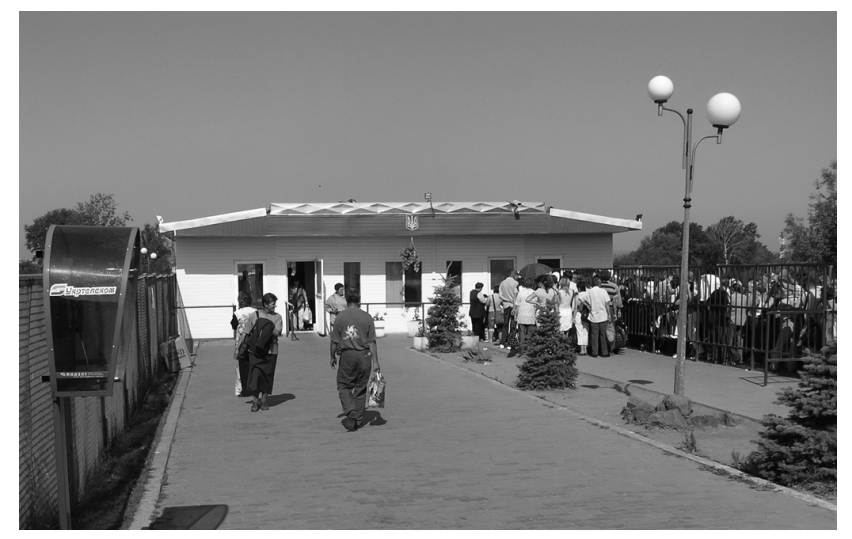

Abbildung 2. Abfertigung von Fußgänger/innen an der polnischenukrainischen Grenze in Medyka (Foto vom Zoll der Ukraine zur Verfügung gestellt).

wohl ein „solches Verhalten sehr unhöflich und unschön“ sei (ebd., Z. 456f.), äußert er aber doch Verständnis dafür, dass den Zöllner/inne/n „schon mal die Nerven durchgehen“, „denn normale Grenzgänger gibt es nur wenige, die Mehrheit sind Schmuggler, mit denen sie da die ganze Zeit dieses und jenes haben“" (ebd., Z. 459-461). Wenn also Ukrainer/innen schlecht behandelt werden, so legt seine Darstellung nahe, dann weil sie an der Grenze vor allem als Schmuggler/innen wahrgenommen werden. Im selben Gespräch bestätigt Dariusz das allgemein verbreitete Duzen „,von allen, egal welchen Geschlechts, welchen Alters oder sonstiges“, sieht aber darin vor allem einen Ausdruck der Macht, die die Zöllner in der Kontrollsituation haben. Auch die Belaruss/inn/en werden nach Auskunft unserer Gesprächspartner/innen vielfach aufgrund ihrer Herkunft schlecht behandelt. Allerdings betonte ein belarussischer Unternehmer in Brest, dass er keineswegs eine Behandlung aufgrund nationaler Stereotype wahrnehme, sondern die Behandlung abhängig vom Motiv des Reisenden sei, das aus seinem Visum ersichtlich werde. Im Gegensatz jedoch zu dem oben zitierten polnischen Unternehmer werden aus seiner Sicht die Einkaufstouristen, also die Kleinhändler, bevorzugt abgefertigt - „Ziel der Reise? Einkaufen. Gut fahr weiter!“ (Fjodor, belarussischer Unternehmer, GD 3, Z. 1231-1235, Zitat Z. 1233f.) -, während er selbst mit einem „Dienstvisum“, wie er es nennt, sein Motiv zusätzlich legitimieren muss.

\subsection{Immer unter Verdacht?}

Ein zweites Set von Zuschreibungen resultiert aus der generellen wie auch aus der situativen Bewertung von Kleinhandel und Kleinhändler/in: Dominieren in der Wahrnehmung die illegalen Aspekte dieser Tätigkeit, also der klandestine Transport und die Falschdeklaration gegenüber dem Zoll, dann ist es wahrscheinlich, dass diese Person erstens als Schmuggler und zweitens unter dem Aspekt der Bedrohung der Sicherheit der EU wahrgenommen wird. Dann greift die 
Gleichsetzung von „Schmuggel“ und „Risiko für die Sicherheit". Schmuggel wird folglich als Verbrechen angesehen oder zumindest in die Nähe desselben gerückt.

Anders sieht es aus, wenn die Tätigkeit als unvermeidliches Mittel zum Lebensunterhalt und als (beinahe) reguläre Arbeit angesehen wird. Dann tritt der illegale Aspekt des Schmuggelns, der in der Regel von den Beteiligten gar nicht geleugnet wird, gegenüber seiner situativ begründeten Legitimität in den Hintergrund. Diese Legitimität wird ,,durch diejenigen hervorgebracht, die von der Grenze (...) finanziell abhängig sind, also z.B. Kleinhändler und Unternehmer" (Bruns und Zichner, 2010:26). Sie wird aber auch durch Schmuggelinfrastrukturen gestützt, die an vielen Orten existieren, wie beispielsweise Verkaufsbuden, die Waren einzig zum Zweck des Schmuggelns verkaufen, aber dennoch offiziell geduldet werden, oder ein Linienbus, ,,der keinem anderen Zwecke als dem Schmuggel dient" (Bruns, 2010b:174). Zuschreibungen von Kategorien und Wertungen von Tätigkeiten sind nicht willkürlich, vielmehr können legitimierende Kontexte eine positive Wertung stützen. Vor allem die Bewertung des Kleinhandels als alternativlose Einnahmequelle im Rahmen einer (allgemeinen) sozio-ökonomischen Krise ist hier relevant. Ebenso kann aber auch eine wechselseitige Abhängigkeit von Grenzpersonal und Handelnden von Bedeutung sein, wenn es familiäre oder nachbarschaftliche Beziehungen gibt. Als legitim wird der Kleinhandel meist dann angesehen, wenn er als alternativlose Erwerbsgrundlage gilt; eine solche Position wird nicht nur von vielen Kleinhändler/innen selbst und von nicht am Handel beteiligten Bewohner/innen der Grenzregion vertreten, sondern auch von Mitarbeiter/innen lokaler Verwaltungen, wie Bruns (2010b, 141ff.) an Beispielen aus polnischen Orten an der Grenze zur Oblast Kaliningrad aufzeigt: Die legitimierende Sichtweise auf Kleinhandel wird allerdings mit unterschiedlichen Begründungen legitimiert. So wird einerseits Kleinhandel als Zeichen von Eigeninitiative und Flexibilität in einer entstehenden Marktwirtschaft geschätzt, andererseits wird darin auch eine Entlastung der Gemeinden von der Zahlung sozialer Hilfen gesehen, deren Höhe ohnehin nicht zur Deckung der Lebensunterhaltkosten ausreichen würde.

Dass Schmuggel als legitime Einkommensquelle angesehen wird, bedeutet jedoch nicht, dass es sich um eine sozial angesehene Tätigkeit handelt, im Gegenteil: Schmuggel bzw. Kleinhandel ist oftmals eine Tätigkeit, zu der „arme Leute“ (Benedikt, rumänischer Unternehmer, GD 6, Z. 1953), die „armen Menschen“ (Kyrilo, ukrainischer Kleinhändler aus Schowkwa, GD 2, Z. 972) oder die ,untere Klasse“ (Benedikt, rumänischer Unternehmer, GD 6, Z. 1884) gezwungen sind. Vielfach sind es Rentner/innen, die wegen nicht ausgezahlter oder sehr niedriger Renten auf einen Zuverdienst angewiesen sind. Von Grenzautoritäten (fälschlicherweise) als Schmuggler/in identifiziert zu werden, wird daher in zweierlei Hinsicht als abwertend empfunden: Zum einen, weil es als ökonomische Tätigkeit aus einer sozialen Notlage resultiert und mit einem niedrigen sozialen Status verbunden wird.
Zum anderen, weil die Person damit in die Nähe von Verbrechern, z.B. von Dieben, gestellt werden kann. Grenzüberschreitend tätige Unternehmer/innen, die selbst nicht oder nicht mehr im Kleinhandel tätig waren, betonten daher den Unterschied zwischen ,,richtigen“ Dienst- und Geschäftsreisen und den Fahrten der Kleinhändler/innen, der eine Differenz in Bezug auf die soziale Hierarchie wie auch auf die Legalität des Tuns darstellt. Das Ineinandergreifen dieser beiden Logiken verdeutlicht ein kurzer Dialog zwischen Unternehmern aus Brest:

Doran: „Besonders unangenehm ist es, wenn man in meiner Tasche nach Zigaretten und Wodka sucht! In meiner Tasche! Ich bin auf Dienstreise! (...) Aber diese polnischen Zollfrauen prüfen meine Tasche. Ich sage: ,Pani, ich habe nichts davon, wonach sie suchen. 'Sie sucht dennoch bei fast jedem nach.“ Fjodor: „Das ist immer so an der Grenze und man wird in jedem Land streng geprüft. Sogar im Geschäft kann man geprüft werden. Aus Verdacht, dass man was geklaut hat. Wichtig ist, dass man dabei höflich behandelt wird, nicht anmaßend.“ Kostja: „Mindestens ,Sorry“ kann man sagen.“ Elian: „Jeder Belarusse oder Russe wird als ein Schmuggler behandelt" (GD 3, Z. 920-932, Herv. JM).

Selbst wenn Schmuggel als legitim gilt, kann sich ein Schmuggler an der Grenze nie sicher sein, dass dies von seinem Gegenüber in einer bestimmten Situation auch so gesehen und über den Schmuggel hinweggesehen wird oder ob er/sie nicht doch als jemand behandelt wird, der/die gegen das Gesetz verstößt. Die Vertreter/innen der Grenzbehörden, insbesondere des Zolls, werden daher ebenfalls stets genau taxiert. Für die Kleinhändler/innen sind dabei zwei Fragen relevant: Treffe ich auf jemanden, der/die auf die Einhaltung der Rechtsvorschriften pocht? Und: Welche Absicht wird mir unterstellt und wie wird sich der/die Zöllner/in und der/die Grenzschutzbeamte/in mir gegenüber verhalten? Einige der Kleinhändler/innen beschäftigte dabei die Frage, auf welchen Annahmen, Beobachtungen und Erwartungen auf Seiten der Vertreter/innen der Grenzbehörden die jeweilige Art des Behandelt-Werdens basiert. Sind es Indizien in der konkreten Interaktion, die eine Behandlung als „Schmuggler“ oder „Verbrecher“ nach sich ziehen, oder handelt es sich um eine Grundeinstellung, wie der Nachsatz von Elian im vorangehenden Zitat nahe legt? Während in einigen Fällen die jeweilige Einordnung aus der unmittelbaren Situation bzw. Interaktion entstanden zu sein scheint, die natürlich auch immer im Kontext früherer Interaktionen zu sehen sind, sehen andere Kleinhändler/innen, in der Weise wie sie behandelt werden, eine pauschale Einordnung, die unabhängig von der konkreten Person oder der konkreten Situation stattfindet. Kyrilo, ein ukrainischer Kleinhändler, berichtet über die Veränderungen an der Grenze zu Polen, nach dem in den 1990er Jahren zunächst ein relativ liberales Grenzregime herrschte: „Danach gab es eine Periode, als jeder, der über die Grenze fuhr, als ein Schmuggler, eine potenziell gefährliche Person betrachtet wurde. Dadurch bekamen die 
Tabelle 1. Übersicht über die im Text verwendeten Gruppendiskussionen.

\begin{tabular}{lll}
\hline Nr. & Teilnehmer/innen & Ort und Datum \\
\hline GD 1 & Kleinhändler/innen aus der Ukraine & Solotvino (UA), 28.7.2008 \\
GD 2 & Kleinhändler/innen aus der Ukraine & Schowkwa (UA), 22.7.2008 \\
GD 3 & Unternehmer/innen aus Belarus & Brest (BY), 14.4.2008 \\
GD 4 & Kleinhändler/innen aus Polen & Przemyśl (PL), 3.6.2008 \\
GD 5 & Kleinhändler/innen aus Polen & Białystok (PL), 9.4.2008 \\
GD 6 & Unternehmer aus Rumänien & Sighetu Marmatiei (RO), 15.5.2008 \\
GD 7 & Unternehmer/innen aus Polen & Przemyśl (PL), 4.6.2008 \\
\hline
\end{tabular}

einfachen Menschen viele Probleme an der Grenze“ (Kyrilo, ukrainischer Kleinhändler, GD 2, Z. 98-101). Diese Phase dauert aus Kyrilos Sicht bis heute an; die Einführung des Schengen-Visums wird von ihm nicht ausdrücklich als zeitliche Markierung betont. Das Klima des Verdachts gegen jeden, der aus der Ukraine kommt und in die Europäische Union einreisen will, wird von einem anderen ukrainischen Kleinhändler, der an der Grenze zu Rumänien lebt, dagegen eindeutig in Verbindung mit dem EU-Beitritt gebracht: „Das Erste, was kommt, das Beleidigendste, man wird beschuldigt, gleich beleidigt - ,Drogen“. Nun wer gibt ihnen das Recht, als Mitglied der EU schon, jeden beliebigen, der die Grenze der EU überquert hat, dass man Drogen transportiert?“ (Basarab, ukrainischer Kleinhändler, GD 1, Z. 843846). Und Filip, ein polnischer Kleinhändler, der in der Nähe der belarussischen Grenze lebt, fühlt sich durch die Art der Durchführung von Hinterland-Kontrollen wie ein Verbrecher behandelt: „Dort wirst du als irgendein Verbrecher betrachtet, gleich knallen sie dir alles an den Kopf. Du sollst aus dem Wagen aussteigen, alles geht kaputt, und du beginnst alles heraus zu nehmen. Findet er etwas, Gott bewahre!, wirst $\mathrm{du}$ in Fesseln gelegt. Ich habe das mit eigenen Augen gesehen." (GD 5, Z. 1767-1772). An diesen Aussagen ist nicht ihr ohnehin nicht überprüfbarer Wahrheitsgehalt interessant, sondern das, was damit zum Ausdruck gebracht wird. Das Empfinden nämlich, grundsätzlich und vor jeder Tat schon in eine bestimmte Kategorie einsortiert worden zu sein: die des ,Schmugglers“ der ,gefährlichen Person“ und des ,,Verbrechers", der dann nicht einmal mehr mit rechtsstaatlichen Mitteln behandelt werden muss. Woher dieses negative Stereotyp stammt, wird nicht angesprochen. Ein Gesprächsteilnehmer versucht, das als empörend empfundene Verhalten der Vertreter des Grenzregimes darauf zurückzuführen, wie die Grenzregelungen derzeit funktionieren und gehandhabt werden. Im Gegensatz zur Intention einer gemeinsamen und homogenen Regelung sieht er vor allem die Heterogenität in der Praxis. Gleichzeitig distanziert er sich und ,seine“ Gruppe, die der „Geschäftsmänner“, von anderen, die die Behandlung ,wie Verbrecher“ möglicherweise zu Recht trifft:

Verschiedene Unklarheiten solcher Art sind die Vorbedingungen für Bestechlichkeit, für Korruption. In der Situation, wenn jedes Land eigene Re- geln im Beantragen des Visums, in der Registrierung der Ladung, in den Formalitäten während der Überschreitung der Grenze hat, führt dies zu Konflikten und zu Korruption. Das muss von der Seite der EU unbedingt gut geregelt werden. Ich hoffe, das passiert bald. Aber momentan ist es noch nicht so, leider. Und wir, die Geschäftsmänner, die alles nach dem Gesetz machen, werden wie Verbrecher behandelt. Genau so. Alle wurden als Verbrecher behandelt (Elian, belarussischer Unternehmer, GD 3, Z. 1218-1230).

\subsection{Paradoxe Konsequenz?}

Die Harmonisierung des europäischen Grenzregimes hat in der Wahrnehmung der Betroffenen paradoxerweise nicht zu einer größeren Klarheit und Transparenz der Regelungen geführt, sondern zu mehr Willkür in der Behandlung des Einzelnen. Manche Teilnehmer/innen bezeichnen dies als ,den menschlichen Faktor", andere benennen die gestiegene Bedeutung der Konsulate, die der Bestechlichkeit und Korruption ihrer Ansicht nach Vorschub leistet. Grenadii, ein belarussischer Unternehmer, geht im selben Gespräch sogar soweit, die Existenz einheitlicher Regeln ganz zu leugnen: „Es gibt“, stellt er kategorisch fest, ,keine gemeinsamen Regeln für die Einreise in die EU. Der Konsul, der Botschafter ist der Zar und der Gott und der Held auf unserem Territorium“ (ebd., Z. 1296-1298). ,Willkür“ und „,menschlicher Faktor“ haben ihren systematischen Grund in der Verlagerung von Identifikation und Reiseerlaubnis in das Vorfeld des Grenzübertritts. Das aber erfordert, aus der Sicht der Kleinhändler/innen und Kleinunternehmer/innen, neue Strategien und Taktiken, insbesondere den Aufbau sozialen Kapitals in Form von persönlichen Kontakten und Netzwerken. Aus dem Kreis derer, die an den Gruppendiskussionen und Interviews teilnahmen, verfügten zum damaligen Zeitpunkt nur wenige über ein solches soziales Kapital. Einige arbeiteten aber gezielt und mit differenzierten Taktiken daran (vgl. Müller und Miggelbrink, 2013).

Zum Zeitpunkt unserer Untersuchungen hatten viele Gesprächsteilnehmer/innen noch keine neuen Strategien entwickelt, sondern vielfach den Kleinhandel aufgegeben. In einem Fall konnte sich eine belarussische Händlerin als 
offizielle Vertriebspartnerin eines polnischen Modeherstellers registrieren lassen und hat so ihren Handel formalisiert. Andere haben vor allem ihr soziales Kapital ausgebaut und sich für alle denkbaren Situationen Empfehlungs- und Beglaubigungsschreiben verschafft, die den situativen Umgang mit dem Grenzpersonal erleichtern (vgl. Müller und Miggelbrink, 2013).

\section{Fazit}

Am Beispiel von Kleinhändler/innen, die über die Außengrenze der Europäischen Union hinweg tätig sind, wurde untersucht, welche Fremd- und Selbstzuschreibungen im Prozess der Grenzüberschreitung relevant werden. Der Kleinhandel an den von uns untersuchten Grenzen ist auf zweifache Weise durch das Schengener Grenzregime beeinflusst worden. Zum einen wurden die Regelungen zur Grenzüberquerung in einer Weise verschärft, die den Kleinhandel unrentabel machen, weil die Hürden der Visumsbeschaffung als zu hoch empfunden werden. Eine bis dahin geduldete ökonomische Praxis zwischen Nachbarstaaten, die im lokalen Kontext oftmals als lebensnotwendig und moralisch gerechtfertigt wahrgenommen wird, wird damit als Nebeneffekt des strikten Grenzregimes eingedämmt. Zum anderen werden die Praktiken des Grenzhandels insbesondere in den Veröffentlichungen der europäischen Grenzschutzagentur FRONTEX zunehmend mit Formen des organisierten Verbrechens assoziiert.

In den individuellen Äußerungen ließen sich Bezugnahmen auf sehr unterschiedliche Kategorien feststellen: Erstens werden Kategorien relevant, die aus den Strukturierungsansprüchen des Schengener Grenzregimes resultieren. Zentral ist hier die Visumspflicht für die Angehörigen bestimmter Staaten, deren Angehörige sich dadurch als auf Distanz gehalten empfinden, obwohl sie sich selbst als Nachbarn und Teilhaber an einer europäischen Identität sehen. Der Strukturierungsakt, der in der Unterscheidung von visumspflichtigen und nicht-visumspflichtigen Staatsangehörigen manifest wird, wird individuell als geopolitisch hierarchisierte oder stratifizierte Identitätszuschreibung erlebt. Aus der Visumspflicht resultiert zudem, dass jede/r Reisende einer individuellen Prüfung unterzogen wird, die aufwändig ist und teils unter unangenehmen und als demütigend empfundenen Bedingungen stattfindet. Da die Praktiken der Visumsvergabe aus der Sicht der Antragsteller/innen vielfach weniger von den abstrakten Reglementierungen als von den Personen abhängen, die darin involviert sind, wird die Wahrnehmung einer hierarchischen Ordnung noch durch die Erfahrung verstärkt, einem willkürlichen System ausgesetzt zu sein.

Zweitens: Weil sich die hier im Zentrum stehenden Kleinhändler/innen in der Grauzone informeller ökonomischer Aktivitäten bewegen, spielt in den Fremdzuschreibungen, die sie erfahren, die Wahrnehmung und Bewertung des Grenzhandels eine zentrale Rolle. Diese sind wiederum von ei- ner Vielzahl von Faktoren abhängig. Einerseits sind solche Strukturierungsakte relevant, die Kleinhandel als zwar illegale, aber tolerierbare ökonomische Aktivität in Krisenzeiten sehen. Andererseits gibt es - insbesondere von Seiten der europäischen Grenzschutzagentur FRONTEX - Strukturierungsakte, die den Kleinhandel in die semantische Sphäre der organisierten Kriminalität einordnen. Zwischen der - mittlerweile zurückgehenden - faktischen Duldung von Kleinhandel als moralisch legitimierte (Über-)Lebensstrategie auf der einen Seite und der semantischen Zuordnung im Rahmen eines versicherheitlichten Grenzregimes entsteht mithin ein zunehmender Widerspruch. Zum Zeitpunkt der Untersuchung äußerte sich dieser in einer zunehmenden Marginalisierung von Kleinhandel und Kleinhändler/innen.

Drittens: Da viele Kleinhändler/innen nicht freiwillig in diesem Metier tätig sind, sondern sich durch äußere Umstände - insbesondere durch eine prekäre Arbeitsmarktlage sowie unzureichende Sozialleistungen und Rentenzahlungen aufgrund ihrer Einkommenssituation dazu gezwungen fühlen, wird der Kleinhandel oft mit einem niedrigen sozialen Ansehen verbunden. Dies äußert sich dann wiederum in abgrenzenden Positionierungen von Unternehmer/innen, die nicht im Kleinhandel tätig sind und daher empört sind, wenn sie als solche angesehen werden. Warum sie (fälschlicherweise) als solche angesehen werden, hängt offensichtlich auch mit immer noch wirksamen stereotypen Verknüpfungen von nationaler Herkunft und sozialer Position ab: Wer aus Belarus kommt, müsse vermutlich ein/e Kleinhändler/in sein und entsprechend behandelt werden.

Das versicherheitlichte Grenzregime strukturiert nicht alle Positionierungen vor, die auf die handelnden Subjekte zugeschrieben werden, mit denen sie sich identifizieren können und die für das Handeln relevant werden. Offensichtlich spielen lang tradierte Wahrnehmungen ,des anderen“ ebenfalls eine wichtige Rolle in der Frage, wie Reisende behandelt und welche Schemata auf sie angewendet werden. Aber es ist festzustellen, dass das Grenzregime sowohl aufgrund seiner derzeitigen Regelungen wie auch in den Praktiken seiner Umsetzung vor Ort, entgegen der politisch oft hoch bewerteten nachbarschaftlichen Beziehungen zu den Staaten und ihren Bevölkerungen, jenseits der einheitlichen Grenze des Schengenraums, Exklusionen vertieft und Antagonismen verschärft hat.

Edited by: O. Söderström

Reviewed by: three anonymous referees

\section{Literatur}

Andreas, P.: Redrawing the line, Int. Security, 28, 78-111, 2003.

Balibar, E.: Europe as borderland, Environ. Plann. D, 27, 190-215, 2009.

Batory Foundation: Gateways to Europe. Checkpoints on the EU External Land Border, Warschau, 2010. 
Bekus-Goncharova, N.: Living in visa territory, Eurozine, http: //www.eurozine.com/articles/2008-02-22-goncharova-en.html (letzter Zugriff: 3. November 2009), 2008.

Belina, B. und Miggelbrink, J.: Am Ostrand des ,wettbewerbsfähigsten Wirtschaftsraums der Welt". (Raum-) Theoretische Überlegungen zur Produktion der EU-Außengrenze als Territorialisierungs- und Skalenstrategie, in: Alltag im Grenzland. Schmuggel als ökonomische Strategie an der EUOstgrenze, Herausgeber: Wagner, M. und Łukowski, W., Wiesbaden, 215-240, 2010.

Belina, B. und Miggelbrink, J.: Risk as a technology of power. FRONTEX as an example of the de-politicisation of EU migration regimes, in: The Spatial Dimension of Risk. How geography shapes the emergence of riskscapes, Herausgeber: Müller-Mahn, D., London, Routledge-Earthscan, 124-136, 2013.

Bohnsack, R.: Rekonstruktive Sozialforschung: Einführung in die Methodologie und Praxis qualitativer Forschung, Opladen, 1999.

Boswell, C.: Migration Control in Europe After 9/11: Explaining the Absence of Securitization, J. Common Mark. Stud., 45, 589610, 2007

Bröckling, U.: Das unternehmerische Selbst, Soziologie einer Subjektivierungsform, Frankfurt/M., 2007.

Brubaker, R. und Cooper, F.: Beyond „identity“, Theor. Soc., 29, 1-47, 2000.

Bruns, B.: „Schwere Arbeit, unsicheres Brot“ - Schmuggel an der polnisch-russischen Grenze als prekäre Erwerbsform, in: Alltag im Grenzland. Schmuggel als ökonomische Strategie an der EUOstgrenze, Herausgeber: Wagner, M. und Łukowski, W., Wiesbaden, 55-72, 2010a.

Bruns, B.: Grenze als Ressource. Die soziale Organisation von Schmuggel am Rande der Europäischen Union, Wiesbaden, 2010b.

Bruns, B. und Zichner, H.: Moral an der Grenze? Theoretische Überlegungen und empirische Befunde zur Moral im Alltag an der östlichen Außengrenze der Europäischen Union, Geographische Revue, 1, 21-36, 2010.

Bruns, B., Miggelbrink, J., und Müller, K.: Smuggling and smallscale trade as part of informal economic practices. Empirical findings from the Eastern external EU border, International Journal of Sociology and Social Policy, 31, 664-680, 2011.

Bruns, B., Müller, K., Wust, A., und Zichner, H.: Grenzüberschreitende ökonomische Praktiken an den östlichen EUAußengrenzen. Der Umgang von Kleinhändlern und Kleinhändlerinnen sowie Unternehmern und Unternehmerinnen mit Grenzregimen, in: Alltag im Grenzland: Schmuggel als ökonomische Strategie im Osten Europas, Herausgeber: Wagner, M. und Łukowski, W., Wiesbaden, 129-146, 2010.

Buckel, S. und Wissel, J.: Entgrenzung der europäischen Migrationskontrolle - Zur Produktion ex-territorialer Rechtsverhältnisse, in: Demokratie in der Weltgesellschaft, Herausgeber: Brunkhorst, H., Baden-Baden, 385-403 (= Soziale Welt, Sonderband 18), 2009

Castells, M. and Portes, A.: World underneath: the origins, dynamics, and effects of the informal economy, in: The Informal Economy. Studies in Advanced and Less Developed Countries, Herausgeber: Castells, M. und Portes, A., Baltimore, 11-37, 1989.

Davies, B. und Harre, R.: Positioning: The Discursive Production of Selves, J. Theor. Soc. Behav., 20, 43-63, 1990.
De Boer, K.: Frontex: Der falsche Adressat für ein wichtiges Anliegen, Kriminol. J., 42, 181-195, 2010.

Donnan, H. und Wilson, T. M.: Territoriality, anthropology, and the interstitial: subversion and support in European borderlands, Focaal - European Journal of Anthropology, 41, 9-20, 2003.

Ein sicheres Europa in einer besseren Welt. Europäische Sicherheitsstrategie, Brüssel, http://consilium.europa.eu/uedocs/ cmsUpload/031208ESSIIDE.pdf, letzter Zugriff: 4. Mai 2012.

Fischer-Lescano, A. und Tohidipur, T.: Europäisches Grenzkontrollregime. Rechtsrahmen der europäischen Grenzschutzagentur FRONTEX, in: Zeitschrift für ausländisches öffentliches Recht und Völkerrecht, 67, 1219-1276, 2007.

Foucault, M.: Sicherheit, Territorium, Bevölkerung: Vorlesung am Collège de France, 1977-1978, Herausgeber: v. Michel Sennelaert, Frankfurt a.M., 2007/1977-1978.

FRONTEX: Eastern Borders Risk Analysis Network, Annual Overview 2011, http://frontex.europa.eu/assets/Attachments_News/ eb_ran_annual_overview_2011_for_public_release.pdf (letzter Zugriff: 3. Mai 2012), 2011 a.

FRONTEX: Futures of Borders. A Forward Study of European Border Checks. O.O., http://frontex.europa.eu/assets/Publications/ Research/Futures_of_Borders.pdf (letzter Zugriff: 4. Mai 2012), $2011 b$.

FRONTEX: Eastern Borders. Annual Overview 2012, Warschau, http://www.frontex.europa.eu/assets/Publications/Risk_ Analysis/EB_AO.pdf (letzter Zugriff: 18. Juni 2013), 2012.

Haase, A., Wust, A., Knappe, E., und Grimm, F.-D.: Wandel in ostmitteleuropäischen Grenzregionen. Auswirkungen der zunehmenden Durchlässigkeit der polnischen Ostgrenze auf Grenzregionen und Grenzbeziehungen, Leipzig, 2004.

Huysmans, J.: The European Union and the Securitization of Migration, J. Common Mark. Stud., 38, 751-777, 2000.

Laclau, E. und Mouffe, Ch.: Hegemony and Socialist Strategy, London, New York, 1985.

Laclau, E. und Mouffe, C.: Hegemonie und radikale Demokratie. Zur Dekonstruktion des Marxismus, Wien, 2006.

Laitinen, I.: Role of Border Control in the new EU security architecture, Vortrag auf dem 14. Europäischen Polizei-Kongress, Berlin, http://www. european-police.eu/Review/binarywriterservlet?imgUid= 4637fc4d-4b49-e213-7341-8217b988f2ee\&uBasVariant $=$ 11111111-1111-1111-1111-111111111111 (letzter Zugriff: 4. Mai 2012), 2011.

Müller, K.: Vor den Toren der Europäischen Union. Handlungsorientierungen ökonomischer Akteure an der östlichen EUAußengrenze, Wiesbaden, Springer VS, 2013.

Müller, K. und Wust, A.: Die Methode der Gruppendiskussion in der Praxis: Forschung am östlichen Rand der Europäischen Union, Forum Qualitative Sozialforschung, im Erscheinen, 2013.

Müller, K. und Miggelbrink, J.: “ The glove compartment half-full of letters" - Informality and cross-border trade at the rims of the Schengen Area, in: The informal post-socialist economy, Herausgeber: Morris, J. und Polese, A., London and New York, Routledge, im Erscheinen, 2013.

Nadel, S. F.: Understanding primitive peoples, Oceania, 26, 159173, 1956.

Neal, A. W.: Securitization and Risk at the EU Border, J. Common Mark. Stud., 47, 333-356, 2009. 
Polese, A.: Who has the right to forbid and who to trade? Making sense of illegality on the Polish-Ukrainian border, in: Subverting Borders. Doing Research on Smuggling and Small-Scale Trade, Herausgeber: Bruns, B. und Miggelbrink, J., Wiesbaden, 21-38, 2012.

Round, J., Williams, C. C., und Rodgers, P.: Everyday tactics and spaces of power: the role of informal economies in post-Soviet Ukraine, Soc. Cult. Geogr., 9, 172-185, 2008.

Schatzki, T. R.: The site of the social. A philosophical account of the constitution of social life and change, Pennsylvania State University Press, University Park, 2002.

Singelnstein, T. und Stolle, P.: Die Sicherheitsgesellschaft, Soziale Kontrolle im 21. Jahrhundert, 3. Aufl., Wiesbaden, 2012.

Smith, A. und Stenning, A.: Beyond household economies: articulations and spaces of economic practice in postsocialism, Prog. Hum. Geogr., 30, 190-213, 2006.

Stammler-Gossmann, A.: "Winter-tyres-for-a-flower-bed": Shuttletrade on the Finnish-Russian border, in: Subverting Borders. Doing Research on Smuggling and Small-Scale Trade, Herausgeber: Bruns, B. und Miggelbrink, J., Wiesbaden, 233-255, 2012 .
Töpfer, E.: Mobile Daten - begrenzte Kontrolle. Auf dem Weg zu einem europäischen Informationsverbund, in: Bürgerrechte \& Polizei/CILIP, 91, 19-32, zitiert nach der online-Ausgabe, http: //www.cilip.de/ausgabe/91/informationsverbund.htm (letzter Zugriff: 31. Januar 2012), 2008.

Torfing, J.: Achievements, Arguments, and Challenges, in: Discourse Theory in European Politics, Herausgeber: Howarth, D. und Torfing, J., Hampshire, New York, 1-32, 2005.

Van Houtum, H. und Pijpers, R.: The European Union as Gated Community: The Two-Faced Border and Immigration Regime of the EU, Antipode, 37, 291-309, 2007.

Wagner, M.: Die moralische Ökonomie des Schmuggels, in: Alltag im Grenzland. Schmuggel als ökonomische Strategie an der EUOstgrenze, Herausgeber: Wagner, M. und Łukowski, W., Wiesbaden, 73-90, 2010.

Walters, W.: Mapping Schengenland: denaturalizing the border, Environ. Plann. D, 20, 561-580, 2002.

Weiss, A.: Crossing conflicting state boundaries: The GeorgianAbkhazian Ceasefire Line, in: Subverting Borders. Doing Research on Smuggling and Small-Scale Trade, Herausgeber: Bruns, B. und Miggelbrink, J., Wiesbaden, 213-232, 2012. 